\title{
Identifying Diseases and Diagnosis using Machine Learning
}

\author{
Iswanto Iswanto, E. Laxmi Lydia, K. Shankar, Phong Thanh Nguyen, Wahidah Hashim, Andino \\ Maseleno
}

\begin{abstract}
The method that is use to optimize the criterion efficiency that depend on the previous experience is known as machine learning. By using the statistics theory it creates the mathematical model, and its major work is to surmise from the examples gave. To take the data straightforwardly from the information the approach uses computational methods. For recognize and identify the disease correctly a pattern is very necessary in Diagnosis recognition of disease. for creating the different models machine learning is used, this model can use for prediction of output and this output is depend on the input that is related to the data which previously used. For curing any disease it is very important to identify and detect that disease. For classify the disease classification algorithms are used. It uses are many dimensionality reduction algorithms and classification algorithms. Without externally modified the computer can learn with the help of the machine learning. For taking the best fit from the observation set the hypothesis is selected. Multi-dimensional and high dimensional are used in machine learning. By using machine learning automatic and classy algorithms can build.
\end{abstract}

Keywords : machine learning; disease detection; computer; classifation algorithm.

\section{INTRODUCTION}

To support the medicinal choices, the ongoing advances in improvements and computing in innovation have encouraged the standard gathering and capacity of medical data. In many nations there is very necessary to store the data of a patient in a proper digital format. For taking the medical decisions that data are then gathered and analyzed [1]. This data include predictions, signal, diagnosis, treatment, image analysis and course. For perceive and distinguish the ailment accurately an example is fundamental in Diagnosis

Revised Manuscript Received on July 22, 2019.

* Correspondence Author

Iswanto Iswanto, Department of Engineer Profession Program, Universitas Muhammadiyah Yogyakarta, Yogyakarta, Indonesia. E-mail: iswanto_te@umy.ac.id

E. Laxmi Lydia, Professor, Vignan's Institute of Information Technology(A),Department of Computer Science and Engineering, Visakhapatnam, Andhra Pradesh, India.

K. Shankar*, Department of Computer Applications, Alagappa University, Karaikudi, India. E-mail: shankarcrypto@gmail.com

Phong Thanh Nguyen*, Department of Project Management, Ho Chi Minh City Open University, Vietnam. E-mail: phong.nt@ou.edu.vn

Wahidah Hashim, Institute of Informatics and Computing Energy, Universiti Tenaga Nasional, Malaysia.

Andino Maseleno, Institute of Informatics and Computing Energy, Universiti Tenaga Nasional, Malaysia. acknowledgment of malady. for making the various models machine learning is utilized, this model can use for expectation of yield and this yield is rely upon the info that is identified with the information which recently utilized. For relieving any sickness it is essential to recognize and distinguish that ailment. For characterize the ailment order calculations are utilized. It uses are numerous dimensionality decrease calculations and grouping calculations [2]. Without remotely adjusted the PC can learn with the assistance of the AI. For taking the best fit from the perception set the speculation is chosen. Multi dimensional and high dimensional are utilized in machine learning. By utilizing machine learning programmed and tasteful calculations can manufacture.

There is lots of scope of machine learning in the field of medical. In some functions like medical imaging, medical knowledge extraction, medical decision support, overall patient management, protein-protein interaction used automatic learning's empirical domain. The machine learning is used for detect and diagnose pneumonia, lung cancer and many other diseases.

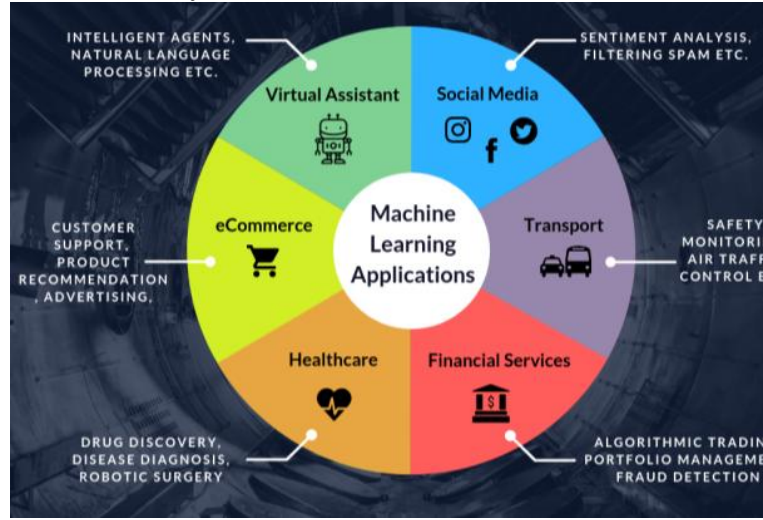

Figure 1: in various fields Machine learning applications

\section{TYPES OF MACHINE LEARNING}

The main two types of machine learning are Supervised are Unsupervised. There is some more machine learning are Semi-Supervised, Deep learning, Evolutionary learning and Reinforcement are discussed below [3]: 


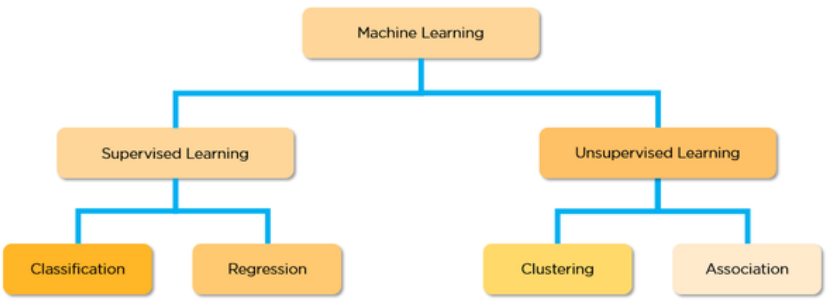

Figure 2: Types of Machine learning

1. Supervised machine learning-

This type of machine learning provides a training data set. As depend on training data set this machine learning method give response accurately to all inputs that are feasible. Supervised Learning is also called as Learning from examples [4]. There are two types of supervised machine learning are regression and classification.

\section{Unsupervised machine learning}

This type of machine learning finds the similarities between the data that is input. On the basis of these similarities, this technique us for data classification. This is often called as [5] estimation of density. on the basis of similarities it have clustering that make clusters.

Some other types of machine learning are:

\section{Semi-supervised machine learning}

This technique is considered as the class of supervised learning techniques. For training this machine learning uses unlabelled data [6]. It is the learning that exists between supervised learning and unsupervised learning. The supervised learning has labeled data and unsupervised learning has unlabelled-data.

\section{Reinforcement machine learning}

Behaviourist psychology supports this type of machine learning. There is using and algorithm that denote that answer is wrong but it does not tell how to correct that answer. Until find the correct answer this algorithm performs many tests [7]. In this learning improvement is not possible.

\section{Evolutionary machine Learning}

This type of learning shows that to make the progress how the biological organisms are adapted [8]. For checking the accuracy of the solution idea of fitness is used.

\section{Deep machine learning}

To optimize the result of the work this learning uses back propagation for study the model parameters [9]. With several processing layers this learning uses deep graph, this graph is made with several transformation that can be linear and nonlinear.

\section{LITERATURE SURVEY}

\section{A. Semantic Relations in Bioscience text}

The paper [10] described that for information extraction structural representation it uses machine learning for mapping the biomedical information. By using text classification the medical abstract is extract. To extract the semantic relation like Gene-Protein from Medline abstracts Naive Bayes (NB) algorithm is used.

\section{B. Learning to extract relations from Medline}

The author of the paper [11] introduced the Naive Bayes classifier processed the individual sentence and it is consider as features. Every feature is obtained as training set of positive attribute. By using CNB algorithm and Naive Bayes, words from Medline abstract the word can be extracting.

\section{Extraction of Disease-Treatment relations from}

Biomedical Sentences

The paper [12] proposed a method in which have between diseases and treatments there are 8 semantic relations is annotated in dataset. For relation identification and entity recognition maximum entropy models and Hidden Markov models are used.

D. Biomedical Language Processing:What's beyond Pubmed The writers of paper [13] show that for processing of biomedical natural language processing is included. By using natural language processing, it takes the disease name and the solution is stored in disease's database.

E. Hybrid Machine Learning Implementation for classifying Disease-Treatment relations in Short texts

The paper [14] presented that by relation identification and sentence selection to identify the most accurate words selection techniques are used. In medical literature the work of relation extraction is performed. in biomedical specific field it uses short texts as data set.

\section{MACHINE LEARNING ALGORITHMS FOR DIAGNOSIS THE DISEASES}

For diagnose the diseases many scientist developed many different machine learning algorithms. The researcher describes that for diagnose the different diseases the machine learning work efficiently. Different approaches used for diagnosis different diseases are shown in figure 2 . There is bunches of extent of machine learning in the field of medicinal. In certain capacities like medicinal imaging, therapeutic information extraction, restorative choice help, by and large patient administration, protein-protein collaboration utilized programmed learning's observational space. The machine learning is utilized for distinguish and analyze pneumonia, lung malignant growth and numerous different ailments. 


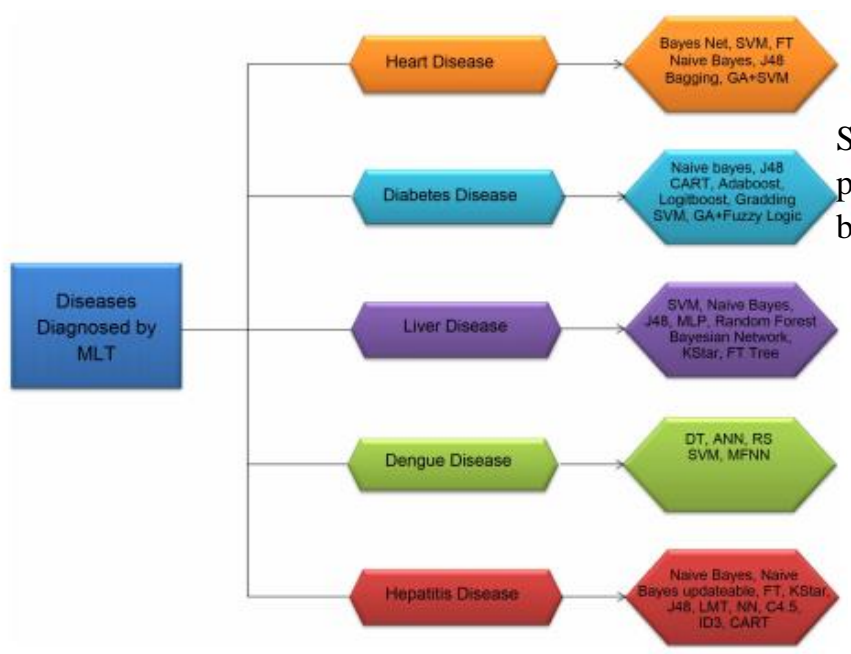

Figure 3: types of diseases that detect by Machine learning [15]

\section{Disease related to heart}

For monitoring and analysis the disease Otoom et al. [16] presented a system. This proposed system is used to monitor and detect the disease called Coronary artery disease. There is taking a data set to find the accuracy of the proposed system. For detect the disease two test are performed. There are using three algorithm Functional Trees, Support vector machine and Bayes Net.

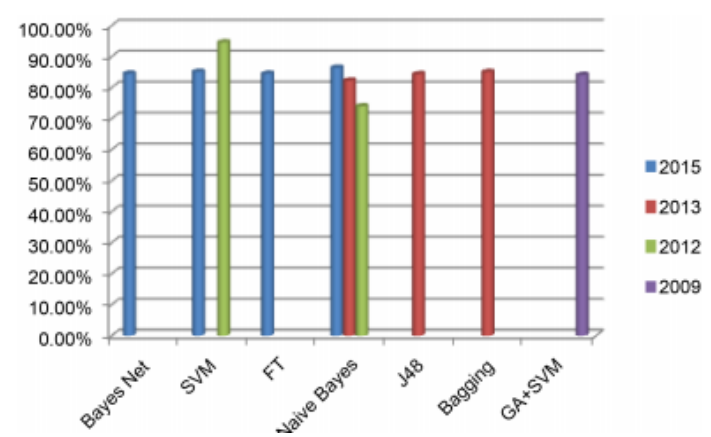

Figure 4: to detect heart disease the accuracy of machine learning [15].

\section{Disease of Diabetes}

By using Naive Bayes and decision tree Iyer et al. [17] introduced a model to detect the disease of diabetes. If there is insulin production in body is insufficient then this disease takes place. By using WEKA data mining tool several test were performed.

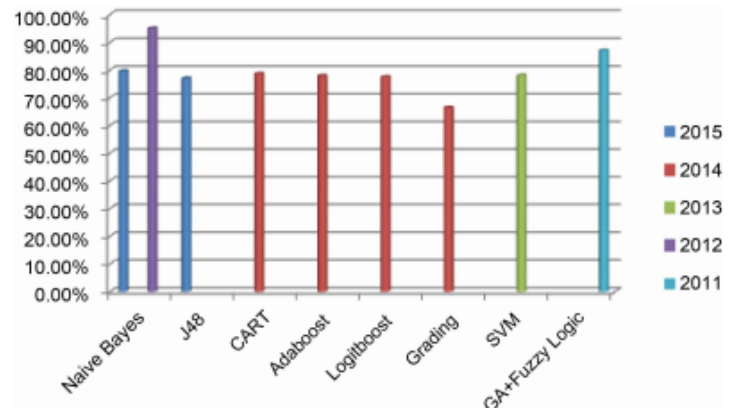

Figure 4 to detect diabetes disease the accuracy of machine learning [15].

\section{Disease of Liver}

By using Naive bayes Classification algorithms and Support vector machine the Vijayarani and Dhayanand [18] predict the disease related to liver. The comparison of data based on time execution and accuracy of the test.

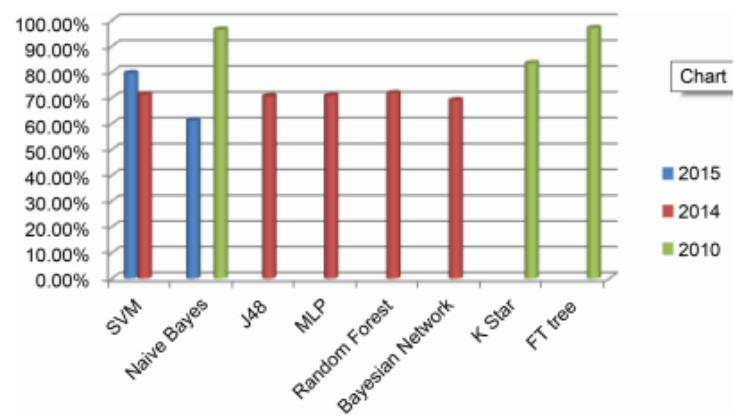

Figure 5: to detect liver disease the accuracy of machine learning [15].

\section{Disease of Dengue}

by using the Data Mining models Tarmizi et al. [19] proposed task for Malaysia Dengue Outbreak Detection. It is a contagious disease. The counties like Malaysia, Indonesia, Thailand, India etc where weather is humid creates some problem. To predict the dengue Artificial Neural Network (ANN), Rough Set Theory (RS) and Decision Tree (DT) are used as classification algorithms.

\section{Disease of Hepatitis}

A comparative analysis is done by Ba-Alwi and Hintaya [20]. Naive Bayes updatable, K Star, NN, J48, LMT, Naive Bayes are the algorithms that use for diagnosis of hepatitis disease. In term of time and accuracy the measurement of classification has done. By using WEKA and neural connections the Comparative Analysis is performed. As compared with result obtain from WEKA the result of neural connections are low.

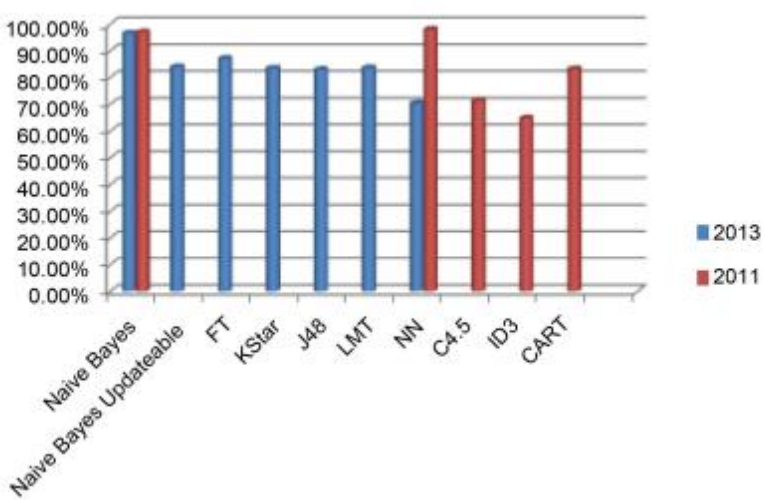

Figure 6: to detect hepatitis disease the accuracy of machine learning[15].

Published By:

Blue Eyes Intelligence Engineering 


\section{Identifying Diseases and Diagnosis using Machine Learning}

\section{CONCLUSION}

The strategy that is use to advance the rule productivity that rely upon the past experience is known as machine learning. By utilizing the measurements hypothesis it makes the scientific model, and its real work is to derive from the models gave. To take the information direct from the data the methodology utilizes computational methods. To bolster the restorative decisions, the progressing propels in upgrades and figuring in advancement has energized the standard social affair and limit of medicinal information. In numerous countries there is extremely important to store the information of a patient in an appropriate advanced arrangement. For taking the therapeutic choices that information are then assembled and investigated. This information incorporates expectations, signal, determination, treatment, picture investigation and course. For check and separate the illness precisely a model is crucial in Diagnosis affirmation of disease.

\section{ACKNOWLEDGEMENT}

This article has been written with financial support of RUSA-Phase 2.0 grant sanctioned vide Letter No. F. 24-51/2014-U, Policy

(TNMulti-Gen), Dept. of Edn. Govt. of India, Dt. 09.10 .2018 .

\section{REFERENCES}

1. Ms.D.Pavithra, Dr.A.N.Jayanthi, "A STUDY ON MACHINE LEARNING ALGORITHM IN MEDICAL DIAGNOSIS", International Journal of Advanced Research in Computer Science,Volume 9, No. 4, July - August 2018.

2. Shaik Razia,P.Swathi Prathyusha,N.Vamsi Krishna,N.Sathya Sumana, "A REVIEW ON DISEASE DIAGNOSIS USING MACHINE LEARNING TECHNIQUES", International Journal of Pure and Applied Mathematics, Volume 117 No. 16 2017, 79-85.

3. D. Yu, and L. Deng, 2011, "Deep learning and its applications to signal and information processing," IEEE Signal Process. Mag., vol. 28, no. 1, pp. 145-154.

4. J. Shi and J. Malik,2000, "Normalized cuts and image segmentation," IEEE Transactions on Pattern Analysis and Machine Intelligence, vol. 22, no. 8, pp. 888-905.

5. A. Kumar, J. Kim, W. Cai, and D. Feng, 2013, "Contentbased medical image retrieval: a survey of applications to multidimensional and multimodality data," J Digit Imaging, vol. 26, no. 6, pp. 1025-1039.

6. N. Tajbakhsh, J. Y. Shin, S. R. Gurudu, R. T. Hurst, C. B. Kendall, M. B.Gotway, and J. Liang, 2016, "Convolutional neural networks for medical image analysis: Full training or fine tuning?" IEEE T Med Imaging, vol. 35, no. 5, pp. 1299- 1312 .

7. Adriana Albu, Loredana Stanciu, 2016, "Benefits of using artificial intelligence in medical predictions," 1EEE, EHealth and Bioengineering Conference (EHB).

8. M. Abedini, N. C. F. Codella, J. H. Connell, R. Garnavi, M. Merler,S. Pankanti, J. R. Smith, and T. Syeda-Mahmood, 2015, "A generalized framework for medical image classification and recognition," IBM J Res Dev, vol. 59, no. 2/3, pp. 1:1-1:18.

9. C. Bishop, 2006, Pattern Recognition and Machine Learning. Springer-Verlag New York.

10. B. Rosario and M.A. Hearst, "Semantic Relations in Bioscience Text," Proc. 42nd Ann. Meeting on Assoc. for Computational Linguistics, vol. 430.

11. M. Craven, "Learning to Extract Relations from Medline," Proc.Assoc. for the Advancement of Artificial Intelligence, 1999.

12. J. Ginsberg, H. Mohebbi Matthew, S.P. Rajan, B. Lynnette, S.S.Mark, and L. Brilliant, "Detecting Influenza Epidemics Using Search Engine Query Data,” Nature, vol. 457, pp. 1012-1014, Feb.2009.

13. L. Hunter and K.B.Cohen, "Biomedical Language Processing:What's beyond PubMed?" Molecular Cell, vol. 21-5, pp. 589-594,2006.
14. M. Goadrich, L. Oliphant, and J. Shavlik, "Learning Ensembles of First-Order Clauses for RecallPrecision Curves: A Case Study in Biomedical Information Extraction," Proc. 14th Int'1 Conf. Inductive Logic Programming, 2004.

15. Fatima, M. and Pasha, M. (2017) Survey of Machine Learning Algorithms for Disease Diagnostic. Journal of Intelligent Learning Systems and Applications, 9, 1-16.

16. Otoom, A.F., Abdallah, E.E., Kilani, Y., Kefaye, A. and Ashour, M. (2015) Effective Diagnosis and Monitoring of Heart Disease. International Journal of Software Engineering and Its Applications. 9, $143-156$. 